\title{
Wnt signaling pathway in retinal vascularization
}

This article was published in the following Dove Press journal:

Eye and Brain

9 August 2016

Number of times this article has been viewed

\section{Kimberly A Drenser}

Associated Retinal Consultants, Royal Oak, MI, USA
Correspondence: Kimberly A Drenser Associated Retinal Consultants, 3535 W Thirteen Mile Road, Suite 344, Royal Oak, MI 48073, USA

Email kdrenser@arcpc.net
Abstract: Wnt-signaling, a ubiquitous pathway that directs differentiation, cell polarity, and tissue specificity, has been implicated as an important gene-expression pathway in retinal development. An increasing body of evidence supports the importance of Wnt-signaling, and specifically, norrin-mediated Wnt-signaling in retinal development and retinal maintenance. Gene mutations affecting the Wnt-signaling pathways result in a variety of inherited vitreoretinopathies. Additionally, there is growing evidence that prematurity and associated retinopathy are associated with alterations in the Wnt-signaling pathways. Further investigations may allow for improved diagnoses, management, and therapies in the future.

Keywords: Wnt, norrin, angiogenesis, development, ROP, prematurity

\section{Background}

Wnt-signaling is a ubiquitous pathway that modulates cellular and tissue differentiation. The Wnt gene family consists of 19 structurally related genes, which encode cysteinerich secreted glycoproteins that act as extracellular signaling factors. Wnt proteins can signal in a variety of ways, depending on the cell type and the receptors and/or coreceptors expressed. Extracellular Wnt ligands interact with their transmembrane Fzd receptors of which there are currently ten identified isoforms in humans, and thereby, activate three diverse signaling pathways. ${ }^{1-4}$ Binding of the Wnt ligand to its Fzd receptor simultaneously with a co-receptor, the low-density LRP5/6, initiates the canonical pathway. This leads to modulation in the transcription of over 50 genes in mammals. ${ }^{1}$

The canonical Wnt/ $\beta$-Catenin pathway is the most thoroughly studied. In the retina, activation is initiated by ligand binding to both the Fzd4 receptor and its co-receptor, LRP5. Multiple Wnt ligands (3a, 5a, 7a, 10a, norrin) are able to bind the Fzd4 receptor. Binding of the receptor complex activates the Dsh protein. This ultimately results in dephosphorylation of $\beta$-Catenin and its subsequent translocation to the nucleus. ${ }^{1,2}$ Nuclear $\beta$-Catenin participates as a transcriptional activator of the TCF/LEF-1 family of DNA binding proteins. Target genes include $c$-myc, CCND1, and VEGF, which presumably regulates cell proliferation in specific tissues.

The planar cell polarity pathway also involves activation of Dsh protein following Fzd4 binding. Unlike the canonical pathway, a co-receptor (LRP5) is not needed for signal activation. One of two small GTPases (Rho or Rac) is thereby activated, which in turn activate an alternative signal transduction pathway. ${ }^{1,2}$ The planar cell polarity 
pathway mediates cytoskeletal organization and cell migration during development and tissue maturation.

The Wnt- $\mathrm{Ca}^{2+}$ pathway (noncanonical signaling) is the least characterized cascade. In the $\mathrm{Wnt}-\mathrm{Ca}^{2+}$ pathway, Fzd receptor activation stimulates an intracellular $\mathrm{Ca}^{2+}$ release, activating CamKII and PKC. This pathway directs cell adhesion and cell movement and is necessary for normal development. Wnt5a is a potent activator of this pathway. ${ }^{3}$

In regards to eye development, a particular Wnt-pathway, norrin-Fzd4, has been identified as playing an essential role in normal retinal angiogenesis. Signaling through the norrin-Fzd4 (canonical and noncanonical) pathways is necessary for the development and maintenance of retinal vasculature. $^{4-7}$ Conversely, increased expression of other Wnt ligands (Wnts 3a, 5a, 7a, and 10a) has been associated with pathologic angiogenesis (neovascularization) in animal models of retinopathy and proliferative diabetic retinopathy. ${ }^{8}$ Without proper activation, dysregulated angiogenesis leads to pathological vascular changes, such as breakdown of tight junctions, capillary drop-out, endothelial budding, and neovascularization, and in extreme cases development of primitive vascular trees (vasculogenesis only). Patients with gene mutations affecting Wnt signaling result in several pediatric vitreoretinopathies, such as Norrie disease, familial exudative vitreoretinopathy (FEVR), and pseudoglioma and osteoporosis syndrome. ${ }^{9-16}$ Additionally, retinopathy of prematurity (ROP) has been associated with gene mutations affecting Wnt signaling. ${ }^{14,17-20}$ Although Coats' disease and persistent fetal vasculature (PFV) are generally unilateral and sporadic, Wnt-pathway mutations have been reported. ${ }^{21-23}$

\section{Retinal vasculogenesis and angiogenesis}

Vasculogenesis is the de novo synthesis of blood vessels. In the human retina the early development of vasculature begins posteriorly, at the optic nerve head. There is clear evidence that vascularization beyond the early stages of vasculogenesis does not occur without norrin-Fzd4 signaling. ${ }^{18}$ Infants with Norrie disease often express mutations in norrin that either eliminates norrin protein translation or affects the tertiary structure, thereby disallowing norrin-Fzd4 binding. Point mutations of the conserved cysteine residues result in improper protein folding. Patients with these mutations have primitive vasculogenesis only and minimal regression of the primary hyaloid structures (Figure 1).

Angiogenesis is the growth of new vessels from existing vessels by way of endothelial budding. When properly controlled, normal retinal development with branching vessels

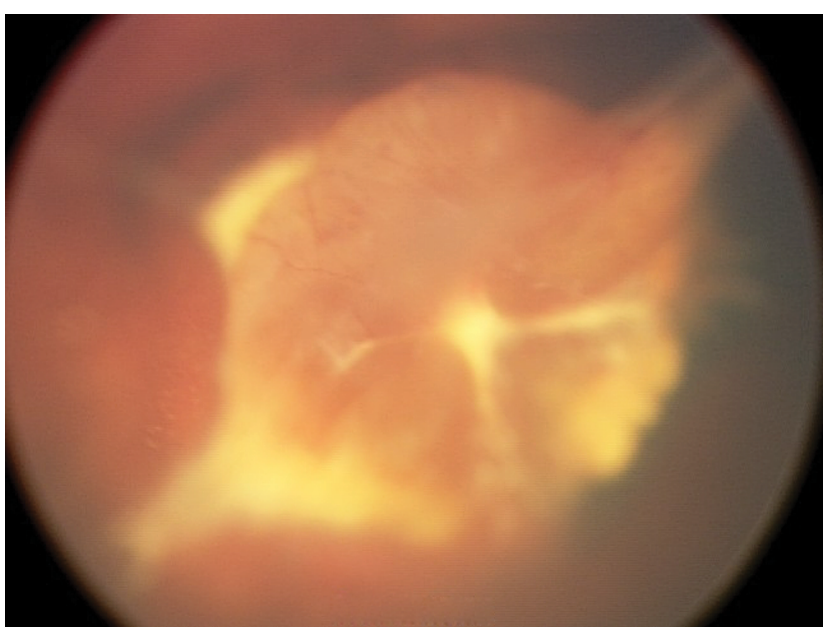

Figure I Example of severe dysgenesis with unregressed stalk tissue and primitive retinal development.

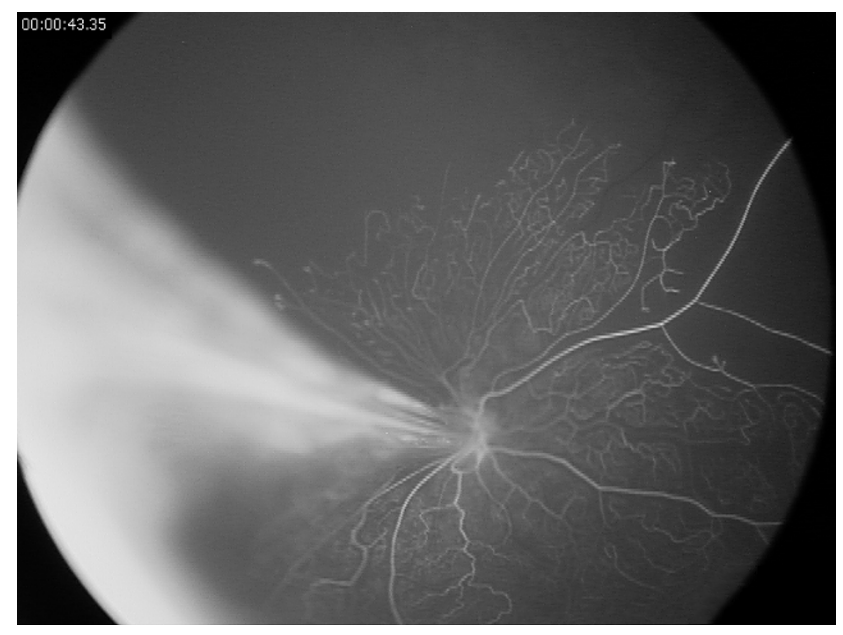

Figure 2 Fluorescein angiogram of a Wnt-associated vitreoretinopathy patient with an Fzd4 mutation.

Note: The avascular periphery, tractional fold, and anomalous capillary formation is noted.

and capillary networks occur. When this is dysregulated, incomplete vascularization and/or pathologic angiogenesis develop (Figure 2). 1,4,7 This can often represent a spectrum of disease severity. In the premature infant, the earliest sign of abnormal angiogenesis is seen as a cessation of retinal vasculature growth. This is primarily an effect of decreased circulating VEGF but is exacerbated by other proangiogenic factors being dysregulated as well. It has been shown in animal models that Wnt pathway activation by norrin is able to overcome this delay in vascular development. ${ }^{24,25}$ Intravitreal injection of norrin following oxygen exposure greatly accelerates the retinal recovery and vascular maturation in the oxygen-induced retinopathy mouse model. ${ }^{24}$ Endogenous expression of norrin in the eye by way of virally transfected lens epithelial cells is protective from oxygen-induced retinopathy and no abnormal retinal development is seen. ${ }^{25}$ 
Norrin may protect the developing eye from abnormal angiogenesis and retinopathy by modulating Wnt signaling as well. A comparison of room air mice and oxygen-induced retinopathy (OIR) mice showed significant changes in the expression of Wnt ligands that bind and activate the Fzd4 receptor. Messenger RNA is significantly increased for Wnts $3 \mathrm{a}, 7 \mathrm{a}$, and 10a but not for norrin during the development of retinopathy ${ }^{26}$ This indicates that norrin provides a protective effect, in part, by modulating the activity of other Wnts when they are pathologically upregulated.

\section{Nonfenestrated vessels}

Retinal vessels are highly specialized nonfenestrated vessels, as seen in the central nervous tissue. This requires vessels to have tight junctions, formed by claudins and occludens, which prevents passive movement of fluids and proteins through the vessel walls. ${ }^{27,28}$

Claudins are integral transmembrane proteins necessary for tight junction formation. ${ }^{28}$ CLD5 is expressed by retinal endothelial cells. At the cellular level, Wnt signaling modulates tight-junction maintenance in the retinal vessels by upregulating expression of CLD5. ${ }^{29-31}$ Disrupted norrinFzd4 binding leads to decreased expression of CLD5 and breakdown of the blood-retinal barrier., ${ }^{426}$ Although there is evidence that norrin activates all three of the intracellular Wnt pathways, ${ }^{32-35}$ loss of the co-receptor LRP5 results in decreased expression of CLD5, indicating that tight junction formation and maintenance is primarily driven by the canonical pathway. ${ }^{26}$

\section{Neurogenesis}

Norrin is a small secreted protein with a cysteine-knot motif. ${ }^{14-16}$ The cysteine-knot motif is highly conserved in many growth factors, including TGF- $\beta$, human chorionic gonadotropin, NGF, and PDGF. The structural similarity of norrin to other growth factors suggests that it may have a function in addition to traditional Wnt-signaling, despite the fact that it is best characterized as a Wnt-receptor ligand. This theory is supported by its lack of structural similarity to other Wnt proteins. ${ }^{17}$

Norrin's role in neurogenesis and neuroprotection is just being elucidated and appears to be multifactorial. Recent investigations have uncovered that norrin acts as a ligand for LGR4, a receptor for Wnt activation that regulates neurogenesis. ${ }^{36}$ Recent mapping of LGR4 expression in the retina shows that it is expressed by Mueller cells, photoreceptor inner segments, retinal ganglion cells, and bipolar cells (Figure 3). This suggests that norrin has multiple roles in

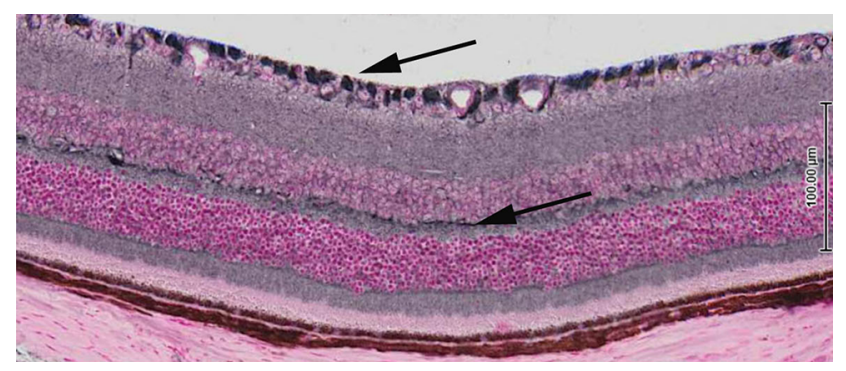

Figure 3 Cross-section of mouse retina post-natal day 21 demonstrating expression of the LGR4 receptor throughout the retina.

Note: Arrows depict higher expression in the retinal ganglion cells and outer plexiform layer.

the formation of the neurovascular unit in the retina. Norrin neuroprotection has been reported in models of retinal toxicity. Seitz et al reported increased survival of retinal ganglion cell (RGC) axons and decreased apoptosis in N-methyl-Daspartate-injected mice following simultaneous injection with norrin. ${ }^{37}$ The authors concluded that norrin's protective effect on RGCs was caused by an increase of Muller cell neurotrophic growth factor expression. Norrin neuroprotection has been demonstrated in the photoreceptors, as well. Braunger and Tamm reported that norrin provided protection against light damage to photoreceptors in mice overexpressing norrin in their RPE cells. ${ }^{5}$ The authors proposed that norrin provides protection to the photoreceptors by increasing the expression of neurotrophic growth factors, specifically BDNF. It is plausible that norrin provides protection to RGCs in OIR eyes by increasing the expression of Muller cell derived neurotrophic factors and, thereby, preventing excitotoxicity and subsequent loss of RGCs.

\section{Clinical manifestations}

To various degrees, eyes with Wnt-associated vitreoretinopathies exhibit incomplete peripheral vascularization, incomplete regression of the primary hyaloid system, capillary drop-out, endothelial budding, and breakdown of the blood-retina barrier, often leading to exudative and tractional retinal detachments. ${ }^{15-18}$ Norrie disease is an X-linked disorder and has the most severe phenotype resulting in blindness, often present at birth. It is often caused by mutations in the conserved cysteine residues of the NDP gene, which comprise the necessary tertiary structure involved in ligandreceptor binding. ${ }^{18}$ In PFV, the premature vasculature of the eye does not regress appropriately, resulting in the formation of a fibrous stalk that extends from the optic disk to the posterior lens capsule. Since the fibrous stalks are common in both PFV and Norrie Disease, the two disorders are often indistinguishable if bilateral disease is present. FEVR occurs 
in X-linked (NDP), autosomal recessive (LRP5), and autosomal dominate inheritance patterns ( Fzd4, TSpan 12, ZNF408). Most reported cases of AdFEVR have been associated with heterozygous mutations in the $F z d 4$ gene. ${ }^{14,16}$ A unifying characteristic in these diseases is an aberration of retinal vascular development demonstrating varying degrees of peripheral avascular retina, abnormal vascularization with retinal neovascularization, and subretinal exudation.

\section{Wnt signaling and ROP}

ROP and FEVR have overlapping phenotypes and an ambiguous birth history may confuse the diagnosis. Generally, ROP is distinguished from FEVR by premature birth and lack of a family history of eye disease. A combination of incomplete peripheral retinal vessel development and exposure to a relatively high oxygen environment (compared to in utero) is believed to set the stage for development of ROP. However, gestational age (GA), birth weight, and systemic health alone do not predict the progression to retinopathy, thus requiring that all at-risk infants have routine screening exams every 1-2 weeks. The overlap in retinal characteristics has supported the hypothesis that a predisposition for ROP is created by genetic alterations. In fact, a twin study demonstrated that there is a likely genetic predisposition for ROP development in $80 \%$ of premature infants with retinopathy. ${ }^{38}$ Mutations in both the NDP and Fzd4 genes have been reported in ROP cases and make Wnt-pathway mutations a likely candidate as a risk factor for developing ROP. ${ }^{14,17-20,39}$

Several studies have reported $F z d 4, N D P$, and $L R P 5$ gene mutations in ROP patients and these appear to be particularly associated with more severe ROP. ${ }^{13-20,30,37}$ The first report discovered mutations located in the 5' untranslated region of the $N D P$ gene ${ }^{18,40}$ A subsequent study found 13\% (7/53) of infants with advanced ROP had either Fzd4 (n=3) or LRP5 (n=4) mutations. ${ }^{17}$ Similarly, Ells et al found three $F z d 4$ mutations (Arg466Trp, Ala370Gly, Lys203Asn) in a group of 71 severe ROP patients and no $\mathrm{Fzd} 4$ mutations in 33 patients with mild or no ROP. ${ }^{20}$ Both studies concluded that $F z d 4$ mutations may have a role in the development or exacerbation of ROP. Ells et al also found the compound variation (P33S;P168S) or either mutation alone in $10 \%$ of their severe ROP patients and in only $3 \%$ of patients with mild to no ROP. However, since they also found (P33S;P168) in 7\% of subjects in a group of random Caucasian samples, they could not make a conclusion regarding the causality of the variation. A recent publication confirmed this finding noting a high association with the Fzd4 compound variation (P33S;P168S) and ROP. In this study, a large patient database $(n=519)$ was analyzed for mutations affecting the Wnt-signaling pathway. ${ }^{41}$ Infants diagnosed with ROP had the highest frequency of the (P33S;P168S) variation (7.5\%) compared to FEVR, possible FEVR, Norrie disease or bilateral PFV. In contrast, the rate in healthy newborns $(3.1 \%)$ was not significantly different from that predicted based on the subjects in the 1,000 Genomes project, with a prevalence of the (P33S;P168S) variation in 20 out of 1,092 random subjects $(1.8 \%)$.

\section{Role of Wnt signaling in fetal development}

The link between premature birth and ROP may lie with altered Wnt signaling. Studies have shown that norrin-Fzd4 signaling affects angiogenesis in the female reproductive system. Markers for angiogenesis and vascular formation are reduced in the corpora lutea of $F z d 4$ null mice and these mice are infertile. ${ }^{42}$ Also, Fzd4 mRNA localizes to vessels and stroma surrounding the mouse embryo. ${ }^{43}$ Similarly, norrin has been localized to the uterine blood vessels and decidual cells of rats, ${ }^{44}$ and NDP knockout mice have defects in vascular development and decidualization in pregnancy that leads to embryonic loss. ${ }^{45}$ In humans, the expression of $N D P$ has been established in the placenta, and $F z d 4$ expression has been localized to placental villous mesenchymal cells. ${ }^{45,46}$

Infants expressing the (P33S;P168S) Fzd4 variant have significantly reduced weight for GA. ${ }^{41}$ The lower than normal birth weights for GA present in the ROP patients with the $(\mathrm{P} 33 \mathrm{~S} ; \mathrm{P} 168 \mathrm{~S})$ variant compared to the other premature infants supports the theory that the Fzd4 receptor is involved in placental formation and/or fetal growth. In fact, five of seven ROP patients with the variation were in the group that had the highest birth weight deficits and were defined as small for gestational age. Presumably, the presence and/or absence of the sequence variation in the mothers would also be a complicating factor in fertility and/or placental development as would additional mutations in NDP; Fzd4 pathway genes. $^{41}$

\section{Summary}

The Wnt signaling pathway plays a critical role in retinal development, in regard to both angiogenesis and neurogenesis. It is also vital for normal fetal growth and may be a predictor for placental insufficiency and preterm birth. Specifically, Wnt activation through proper norrin:Fzd4 binding is specific to retinal development. Alterations affecting this pathway often result in Wnt-associated vitreoretinopathies and may be a risk factor for developing ROP. Therefore, it may be beneficial to screen premature infants for the presence of Wnt signaling mutations so that these patients may 
be monitored more carefully. Additionally, patients with anomalous vasculature and displaying any of the characteristics commonly seen with Wnt-pathway mutations should be offered genetic testing.

\section{Disclosure}

The author has no conflicts of interest to disclose.

\section{References}

1. Tsaousi A, Mill C, George SJ. The Wnt pathways in vascular disease: lessons from vascular development. Curr Opin Lipidol. 2011;22(5): 350-357.

2. Bowerman B. Cell signaling. Wnt moves beyond the canon. Science. 2008;320(5874):327-328.

3. Kühl M. The WNT/calcium pathway: biochemical mediators, tools and future requirements. Front Biosci. 2004;9:967-974.

4. Wang Y, Rattner A, Zhou Y, et al. Norrin/Frizzled4 signaling in retinal vascular development and blood brain barrier plasticity. Cell. 2012; 151(6):1332-1344.

5. Braunger BM, Tamm ER. The different functions of Norrin. Adv Exp Med Biol. 2012;723:679-683.

6. Ohlmann A, Tamm ER. Norrin: molecular and functional properties of an angiogenic and neuroprotective growth factor. Prog Retin Eye Res. 2012;31(3):243-257.

7. Ye X, Wang Y, Nathans J. The Norrin/Frizzled4 signaling pathway in retinal vascular development and disease. Trends Mol Med. 2010;16(9): 417-425.

8. Chen J, Stahl A, Krah NM, et al. Wnt signaling mediates pathological vascular growth in proliferative retinopathy. Circulation. 2011;124(17): 1871-1881.

9. Sims KB. NDP-related retinopathies. In: Pagon RA, Adam MP, Ardinger $\mathrm{HH}$, et al, editors. GeneReviews(R). Seattle (WA): University of Washington, Seattle; 1993.

10. Chamney S, McLoone E, Willoughby CE. A mutation in the Norrie disease gene (NDP) associated with familial exudative vitreoretinopathy. Eye (Lond). 2011;25(12):1658.

11. Chen ZY, Battinelli EM, Fielder A, et al. A mutation in the Norrie disease gene (NDP) associated with X-linked familial exudative vitreoretinopathy. Nat Genet. 1993;5(2):180-183.

12. Dickinson JL, Sale MM, Passmore A, et al. Mutations in the NDP gene: contribution to Norrie disease, familial exudative vitreoretinopathy and retinopathy of prematurity. Clin Experiment Ophthalmol. 2006; 34(7):682-688.

13. Chung BD, Kayserili H, Ai M, et al. A mutation in the signal sequence of LRP5 in a family with an osteoporosis-pseudoglioma syndrome (OPPG)-like phenotype indicates a novel disease mechanism for trinucleotide repeats. Hum Mutat. 2009;30(4):641-648.

14. Drenser KA, Dailey W, Vinekar A, et al. Clinical presentation and genetic correlation of patients with mutations affecting the FZD4 gene. Arch Ophthalmol. 2009;127(12):1649-1654.

15. Gilmour DF. Familial exudative vitreoretinopathy and related retinopathies. Eye (Lond). 2015;29(1):1-14.

16. Toomes C, Downey L. Familial exudative vitreoretinopathy, autosomal dominant. In: Pagon RA, Adam MP, Ardinger HH, et al, editors GeneReviews(R). Seattle (WA): University of Washington, Seattle; 1993.

17. Kondo H, Kusaka S, Yoshinaga A, et al. Genetic variants of FZD4 and LRP5 genes in patients with advanced retinopathy of prematurity. Mol Vis. 2013;19:476-485.

18. Wu WC, Drenser K, Trese M, Capone A Jr, Dailey W. Retinal phenotypegenotype correlation of pediatric patients expressing mutations in the Norrie disease gene. Arch Ophthalmol. 2007;125(2):225-230.
19. Hiraoka M, Takahashi H, Orimo H, et al. Genetic screening of Wnt signaling factors in advanced retinopathy of prematurity. Mol Vis. 2010; 16:2572-2577.

20. Ells A, Guernsey DL, Wallace K, et al. Severe retinopathy of prematurity associated with FZD4 mutations. Ophthalmic Genet. 2010;31(1): $37-43$.

21. Robitaille JM, Wallace K, Zheng B, et al. Phenotypic overlap of familial exudative vitreoretinopathy (FEVR) with persistent fetal vasculature (PFV) caused by FZD4 mutations in two distinct pedigrees. Ophthalmic Genet. 2009;30(1):23-30.

22. Black GC, Perveen R, Bonshek R, et al. Coats' disease of the retina (unilateral retinal telangiectasis) caused by somatic mutation in the NDP gene: a role for norrin in retinal angiogenesis. Hum Mol Genet. 1999; 8(11):2031-2035.

23. Robitaille JM, Zheng B, Wallace K, et al. The role of Frizzled-4 mutations in familial exudative vitreoretinopathy and Coats disease. $\mathrm{Br} \mathrm{J}$ Ophthalmol. 2011;95(4):574-579.

24. Tokunaga CC, Mitton KP, Dailey W, et al. Effects of anti-VEGF treatment on the recovery of the developing retina following oxygen-induced retinopathy. Invest Ophthalmol Vis Sci. 2014;55(3):1884-92.

25. Ohlmann A, Seitz R, Braunger B, et al. Norrin promotes vascular regrowth after oxygen-induced retinal vessel loss and suppresses retinopathy in mice. $J$ Neurosci. 2010;30(1):183-93

26. Chen J, Stahl A, Krah NM, et al. Retinal expression of Wnt-pathway mediated genes in low-density lipoprotein receptor-related protein 5 (Lrp5) knockout mice. PLoS One. 2012;7(1):e30203

27. Haseloff RF, Dithmer S, Winkler L, et al. Transmembrane proteins of the tight junctions at the blood-brain barrier: structural and functional aspects. Semin Cell Dev Biol. 2015;38:16-25.

28. Van Itallie CM, Anderson JM. Claudin interactions in and out of the tight junction. Tissue Barriers. 2013;1(3):e25247

29. Koto T, Takubo K, Ishida S, et al. Hypoxia disrupts the barrier function of neural blood vessels through changes in the expression of claudin- 5 in endothelial cells. Am J Pathol. 2007;170(4):1389-97.

30. Luo Y, Xiao W, Zhu X, et al. Differential expression of claudins in retinas during normal development and the angiogenesis of oxygen-induced retinopathy. Invest Ophthalmol Vis Sci. 2011;52(10):7556-64.

31. Shen W, Li S, Chung SH, et al. Tyrosine phosphorylation of VE-cadherin and claudin-5 is associated with TGF- $\beta 1$-induced permeability of centrally derived vascular endothelium. Eur J Cell Biol. 2011;90(4): 323-32.

32. Descamps B, Sewduth R, Tojais N, et al. Frizzled4 regulates arterial network organization through noncanonical Wnt/Planar Cell polarity signaling. Cir Res. 2012;47-58.

33. Yao R., Natsume Y, Noda T. MAGI-3 is involved in the regulation of the JNK signaling pathway as a scaffold protein for frizzled and Ltap. Oncogene. 2004; 23:6023-6030

34. Robitaille J, MacDonald ML, Kaykas A, et al. Mutant frizzled-4 disrupts retinal angiogenesis infamilial exudative vitreoretinopathy. Nat Genet. 2002;32:326-330.

35. Junge H, Yang S, Burton J, et al. TSPAN12 Regulates retinal vascular development by promoting Norrin - but not Wnt-induced FZD4/ $\beta$ Catenin signaling. Cell. 2009;139:299-311.

36. Deng C, Reddy P, Cheng Y, et al. Multi-functional norrin is a ligand for the LGR4 receptor. J Cell Sci. 2013;126(Pt 9):2060-8.

37. Hutcheson KA, Paluru PC, Bernstein SL, et al. Norrie disease gene sequence variants in an ethnically diverse population with retinopathy of prematurity. Mol Vis. 2005;11501-8

38. Bizzarro MJ, Hussain N, Jonsson B, et al. Genetic susceptibility to retinopathy of prematurity. Pediatrics. 2006;118(5):1858-63.

39. Mohamed S, Schaa K, Cooper ME, et al. Genetic contributions to the development of retinopathy of prematurity. Pediatr Res 2009;65(2):193-7.

40. Hiraoka M, Berinstein DM, Trese MT, et al. Insertion and deletion mutations in the dinucleotide repeat region of the norrie disease gene in patients with advanced retinopathy of prematurity. J Hum Genet. 2001; 46(4):178-81. 
41. Dailey WA, Gryc W, Garg PG, et al. Frizzled-4 variations associated with retinopathy and intrauterine growth retardation: A potential marker for prematurity and retinopathy. Ophthalmology. 2015;122(9): 1917-23.

42. Milhem RM, Ben-Salem S, Al-Gazali L, et al. Identification of the cellular mechanisms that modulate trafficking of frizzled family receptor 4 (FZD4) missense mutants associated with familial exudative vitreoretinopathy. Invest Ophthalmol Vis Sci. 2014;55(6):3423-31.

43. 43.Hsieh M, Boerboom D, Shimada M, et al. Mice null for Frizzled4 (Fzd4-/-) are infertile and exhibit impaired corpora lutea formation and function. Biol Reprod. 2005;73(6):1135-46.
44. Hayashi K, Erikson DW, Tilford SA, et al. Wnt genes in the mouse uterus: Potential regulation of implantation. Biol Reprod. 2009;80(5):989-1000.

45. Kaloglu C, Cesur I, Bulut HE. Norrin immunolocalization and its possible functions in rat endometrium during the estrus cycle and early pregnancy. Dev Growth Differ. 2011;53(7):887-96.

46. Luhmann UF, Meunier D, Shi W, et al. Fetal loss in homozygous mutant norrie disease mice: A new role of norrin in reproduction. Genesis. 2005; 42(4):253-62.

47. Knofler M, Pollheimer J. Human placental trophoblast invasion and differentiation: A particular focus on wnt signaling. Front Genet. 2013;4: 190 .

\section{Publish your work in this journal}

Eye and Brain is an international, peer-reviewed, open access journal focusing on clinical and experimental research in the field of neuroophthalmology. All aspects of patient care are addressed within the journal as well as basic research. Papers covering original research, basic science, clinical and epidemiological studies, reviews and evaluations,

Submit your manuscript here: https://www.dovepress.com/eye-and-brain-journal
Dovepress

guidelines, expert opinion and commentary, case reports and extended reports are welcome. The manuscript management system is completely online and includes a very quick and fair peer-review system, which is all easy to use. Visit http://www.dovepress.com/testimonials.php to read real quotes from published authors. 